Glauert, A. M. \& Brieger, E. M. (1955). J. gen. Microbiol. 13, 310-317

\title{
The Electron-dense Bodies of Mycobacterium phlei
}

\author{
By AUDREY M. GLAUERT* and E. M. BRIEGER
}

Strangeways Research Laboratory and Papworth Hospital, Cambridge

\begin{abstract}
SUMMARY: A method is described in which identical groups of micro-organisms are viewed first in the light microscope and then in the electron microscope. A direct comparison was made between electron micrographs and preparations stained with specific metachromatic, chromatinic and mitochondrial stains. In a study of the electron-opaque granules of the mycobacteria it was shown that they contain metaphosphates and show metachromasia. They are situated between the chromatinic bodies and cannot be identified with mitochondria.
\end{abstract}

There is still considerable controversy concerning the nature of the electronopaque granules which are found in the mycobacteria and other microorganisms. It was realized that more specific information concerning these structures could be obtained by a comparison of identical organisms viewed under different experimental conditions. By mounting the bacteria on electron microscope grids it was possible to photograph groups of organisms in the light microscope and then locate the same groups in the electron microscope. In this way stained preparations and electron micrographs were compared directly, and preparations stained by different procedures were compared among themselves. This method has provided useful evidence concerning the dense bodies of the mycobacteria and has also thrown light on the problem of the bacterial nucleus.

\section{METHODS}

The strain of Mycobacterium phlei (Söhngen) used in these experiments was obtained from the National Collection of Type Cultures in 1943 and has been subcultured subsequently at the Strangeways Laboratory. It is particularly suitable for a study of internal structure owing to its large size, ease of growth and formation of abundant dense bodies under normal cultural conditions. Throughout the present experiments the bacteria were grown on plates consisting of equal parts of glycerol agar and embryo extract.

The comparative microscope methods were carried out with the bacilli mounted on 'formvar' films covering electron microscope grids. Films strong enough to withstand the various staining processes were formed from a $0 \cdot 8 \%$ solution of formvar in ethylene dichloride cast on water. There was a tendency for the formvar films to float off the grids in some of the solutions, but this was prevented by coating the grids with a thin layer of polybutene before picking up the formvar films. When a grid is dipped in a $1.0 \%$ solution of polybutene in xylene and placed on filter-paper to dry, a thin sticky layer forms over the grid bars without obscuring the holes (Drummond, 1950). Flat copper grids (Smethurst High-Light Ltd.) were used to give an even field for light microscopy.

* Sir Halley Stewart Research Fellow. 
To mount the organisms on a grid a square of the inoculated glycerol agar + embryo extract plate was cut out and placed on a glass slide in a Petri dish. A print was taken on the grid by inverting it, with the formvar film downwards, on the growth of organisms on the surface of the plate; when the grid was removed a proportion of the organisms remained attached to the formvar film. When the plate was rather soft some of the medium also stuck to the grid but was easily removed by gentle washing in distilled water. The organisms were fixed by placing the grid face upwards on several layers of filter-paper soaked in $\mathbf{5 \cdot 0} \%(\mathrm{v} / \mathrm{v})$ formalin for $\mathbf{3 0} \mathrm{min}$. Sufficient fixative diffused through the holes in the formvar film. After fixation the grids were washed in distilled water and placed on filter-paper to dry. The organisms could then be viewed in the electron microscope, with or without previous metal shadowing.

When the organisms were stained, the grid was treated like a coverslip preparation and carried through the various staining, washing and differentiating solutions. Specific metachromatic, chromatinic and mitochondrial stains were used.

To make a direct comparison of light- and electron-microscope pictures of identical organisms, the stained grid was mounted in cedar wood oil between a thin slide and coverslip so that the high-power oil-immersion objective of a light microscope could be used. The central four squares of the copper grids were marked and were easily found. Groups of stained organisms lying in these or adjacent squares were photographed on $35 \mathrm{~mm}$. film and enlargements made from the negatives. The grid was then soaked in xylol to remove the cedarwood oil, and after drying was ready for examination in the electron microscope. The central squares were again viewed, and with the help of photographs, already prepared, the same groups of organisms were found with little difficulty and photographed again with the electron microscope. The electron micrographs were taken on a Siemen's electron microscope in the Cavendish Laboratory, Cambridge, at a primary magnification of $c . \times 8000$.

A similar procedure was followed when comparing results with two different stains. After being stained for the first time and photographed, the organisms on the grid were decolorized and restained by the second procedure. Again the same group of organisms was easily found and re-photographed.

\section{RESULTS}

\section{Metachromatic stains}

Bacteria on grids were stained by the standard procedure with Loeffler's methylene blue. In the light microscope a normal $24 \mathrm{hr}$. culture was seen to consist of groups of rods and long filaments in the stage of filamentous proliferation (Brieger, Cosslett \& Glauert, 1954). The organisms were stained blue and had bright red granular inclusions. These red metachromatic granules varied considerably in size, and some of them had an admixture of blue colour so that they appeared red-purple or black.

In the electron microscope the organisms were seen to contain well-defined electron-opaque bodies which corresponded with the metachromatic granules 
in number and position. When identical groups of organisms were viewed, first in the light microscope and then in the electron microscope, it was found that the red granules and the electron-opaque bodies occupied exactly the same positions ( $\mathrm{Pl}$. 1, fig. $1 a, b)$. In the light microscope photograph the red granules have been marked with arrows.

Confirmatory evidence for the metachromatic nature of the dense bodies was given by Neisser's stain for volutin. Again there was a direct correlation between the volutin granules of the stained preparations and the electrondense bodies (Pl. 1, fig. 2a, b). The granules were not so clearly seen as with methylene-blue staining because of the greater density of the cytoplasmic staining which masked other structures. The granules failed to stain metachromatically with toluidine blue.

\section{May-Grünwald and Giemsa stains}

In preparations of the mycobacteria stained with the May-Grünwald stain alone a striking arrangement of red granules was seen within the pale blue rods and filaments. The red bodies could be identified with the electron-opaque granules by a comparison of light and electron-microscope pictures (Pl. 1, fig. $3 a, b)$. The vacuolated appearance of the cytoplasm in the electron micrograph is typical of these bacteria at certain stages of development, and in this organism the effect was enhanced by electron bombardment.

With the complete May-Grünwald and Giemsa stain the red granules were still present in the rods and filaments. In some parts of the preparations they were clearly seen (Pl. 1, fig. $4 a$ ), but in other areas the background staining of the cytoplasm was so intense that the red bodies and other internal structures were barely visible. They could be made more evident by the use of appropriate filters or by allowing the overlying stain to fade. In the electron microscope most of the organisms appeared very dense (PI. 1, fig. $4 b$ ), but they could be made more transparent by electron bombardment. After this treatment the electron-opaque granules were once more apparent, and comparison with the corresponding light-microscope photographs confirmed that they occupied the same positions as the red granules (Pl. 1, fig. 4a,c).

\section{Chromatinic stains}

The organisms on an electron-microscope grid were stained by the HClGiemsa method (Piekarski, 1937; Brieger \& Robinow, 1947), and the familiar picture of rows of irregularly shaped chromatinic bodies was obtained. In the electron microscope the organisms appeared very dense owing to the Giemsa stain, and the electron-opaque granules were either extracted or decreased in density by the hydrolysis procedure. A comparison of light and electron microscope photographs (Pl. 1, fig. 5a,b) indicated some parallel between the chromatinic structures and the denser areas in the electron micrographs, but it can be assumed that at least part of the density was due to the deposition of stain at these sites.

It was noticed that in preparations stained with methylene blue, the blue structures which lay between the red granules resembled the chromatinic 
bodies of the HCl-Giemsa stain. A direct comparison of identical groups of organisms stained by the two methods showed that the 'blue' structures and the chromatinic bodies were exactly the same in shape and position and that the metachromatic granules were no longer visible in the HCl-Giemsa preparations (Pl. 2, fig. 6a,b). The granules which had stained red-purple or black with methylene blue were still present after hydrolysis and presumably only the red-staining substance had been removed from them. One of these granules can be seen in Pl. 2, fig. 6a, and is marked with a double arrow.

\section{Mitochondrial stains}

The location of oxidative-reductive sites in the mycobacteria was studied with triphenyltetrazolium chloride (TTC) and Janus Green B. After 24 hr. of growth on the surface of a glycerol agar + embryo extract plate, prints were taken on formvar-covered electron microscope grids in the usual way. These grids were then placed face downwards on several layers of filter-paper soaked in a glucose + phosphate medium containing $0.01 \%(\mathrm{w} / \mathrm{v})$ TTC or $0.001 \%$ $(\mathrm{w} / \mathrm{v})$ Janus Green B. The preparations were incubated at $37^{\circ}$ and grids removed at various times, mounted without fixation in a drop of glucose + phosphate medium and observed in the light microscope.

After $1 \frac{1}{2}$ hr. incubation coloured granules were observed in some of the TTC preparations and were presumed to represent deposits of the red formazan formed by reduction of TTC. The colour was faint and the granules could only be observed and photographed with the diaphragm of the condenser stopped down considerably. As with the other stains, identical groups of organisms were viewed in the electron microscope and a comparison was made (Pl. 2, fig. $7 a, b)$. In some of the organisms the reduction sites and the electron-opaque granules occupied the same positions but the stained granules were frequently much larger than the dense bodies. Sometimes the deposits of dye were unaccompanied by any corresponding dense granules. No similar stained granules were observed in the mycobacteria grown in contact with Janus Green B even after several hours of incubation. By careful observation a few small peripheral particles were seen but they did not correspond in number or position to the electron-opaque bodies.

\section{Results of treatment with ribonuclease and deoxyribonuclease}

Samples of ribonuclease and deoxyribonuclease (obtained from the Nutritional Biochemical Corporation, Cleveland, Ohio, U.S.A.) were made up as $\mathbf{0} \cdot \mathbf{1} \%(\mathrm{w} / \mathrm{v})$ solutions in glass-distilled water. The deoxyribonuclease solution was further diluted with an equal volume of $0 \cdot 15 \mathrm{M}-\mathrm{MgSO}_{4}$ before use. Treatment of the organisms on an electron-microscope grid with either ribonuclease or deoxyribonuclease for $1 \mathrm{hr}$. at $37^{\circ}$ failed to remove the electron-opaque granules from most of the organisms (Pl. 2, figs. 8, 9). Similarly, the metachromatic granules and the red bodies of the May-Grünwald preparations were unaffected. In one group of organisms the dense granules were differentiated into a darker cortex and lighter core but this was considered to be 
the effect of electron bombardment and not of the action of ribonuclease. Treatment with ribonuclease followed by deoxyribonuclease also failed to remove the granules.

\section{Treatment with perchloric acid and trichloroacetic acid}

Extraction of the organisms with cold $10 \%$ perchloric acid (a $60 \%, \mathrm{w} / \mathrm{w}$, solution diluted with distilled water) for $24 \mathrm{hr}$. caused the disappearance of many of the dense bodies from the electron micrographs (PI. 2, fig. 10), but in a few organisms a resistant cortex of dense material remained. A similar extraction with cold $10 \%(\mathrm{w} / \mathrm{v})$ trichloroacetic acid also removed the dense bodies (Pl. 2, fig. 11). There was parallel disappearance of metachromatic and red granules from the stained preparations.

\section{DISCUSSION}

Earlier observations on the internal structures of mycobacteria viewed in the electron microscope were summarized by Brieger, et al. (1954). The location and development of the electron-opaque granules during reproduction were studied, and it was concluded that they were not the nuclei. The results obtained with the method described in the present paper indicate more specifically the nature of the dense bodies. Observations with methylene blue and the Neisser stain identify these electron-dense bodies with the metachromatic volutin granules revealed by these techniques. Similar results were obtained by Winkler (1953) with a bacillus, and by Smith, Wilkinson \& Duguid (1954) with Aerobacter aerogenes. It was concluded by these authors that the electron opaqueness and staining characteristics of the dense bodies is due to their content of metaphosphates. A similar interpretation for the dense bodies of the mycobacteria is supported by the work of Winder \& Denneny (1954), who found a direct correlation between the amount of metaphosphate present and the number and size of the metachromatic granules in Mycobacterium smegmatis and in $\boldsymbol{M}$.tuberculosis. The extraction of the granules by perchloric acid and trichloroacetic acid also supports this interpretation as suggested by Grula \& Hartsell (1954) for Caulobacter vibrioides.

Although it seems certain that the most striking properties of the electrondense bodies of the mycobacteria are due to their metaphosphate content they also contain other substances. The quantity of metaphosphate present in the cells is not sufficient to account for the total volume of the dense bodies (Smith, et al. 1954), and it seems likely that they also contain ribonucleoprotein, organic material and other substances. Winder \& Denneny (1954) noted that it was difficult to extract the metaphosphate separately from the ribonucleic acid, and suggest that they are bound in a similar manner. However, the fact that the density and staining characteristics of the granules was unaffected by ribonuclease indicates that they did not contain more than small amounts of ribonucleoprotein. When the granules are extracted by acids or volatilized in the electron beam they frequently differentiate into a darker cortex and lighter core, which suggests that a residue of organic material remains. 
In one of the earliest interpretations of the electron-dense bodies of the mycobacteria it was suggested that they are nuclei (Knaysi, Hillier \& Fabricant, 1950; Knaysi, 1951). However, this interpretation has always seemed doubtful to the present authors because of the behaviour of the dense bodies during reproduction. As noticed by other workers also (Winkler, 1953; Takeya, Koike, Uchida, Inoue \& Nomiyama, 1954), the dense bodies do not appear to be essential for the development of the organisms and groups of cells containing no granules reproduce in the normal way. The results described in the present paper with the $\mathrm{HCl}$-Giemsa stain prove that the chromatinic bodies of the mycobacteria examined are not identical with the dense bodies and lie between them (Pl. 2, fig. 6a, $b$ ). A similar result was reported by Tronnier (1953) for Corynebacterium diphtheriae.

Observations with mitochondrial stains show how misleading these procedures can be when applied to bacteria. Some parallel can be drawn between reduction sites and the positions of the electron-opaque granules, but in general the deposits of dye are far larger than the dense bodies (Bielig, Kausche \& Haardick, 1952). The impression is gained that the dense bodies merely act as centres around which the reduced dye is deposited. This interpretation is supported by the fact that in some areas dense bodies are seen with no accompanying stained granules. It was observed by Weibull (1953) that the red formazan resulting from the reduction of triphenyltetrazolium chloride in Bacillus megaterium first appeared as a number of scattered particles and then coalesced to form the familiar granular deposits; it is possible that a similar process occurs in the mycobacteria used here. As long ago as 1951 Wallhäusser reported the movement of reduction sites within living cells; these observations throw grave doubts on the suggestion that the electrondense bodies represent the mitochondria of bacterial cells (Mudd, 1953).

It was hoped that the application of the May-Grünwald and Giemsa stain to the mycobacteria would provide the same clear differentiation of ribonucleoprotein and deoxyribonucleoprotein as obtained in mammalian cells (Jacobson $\& W e b b, 1952)$, in which the ribonucleoprotein stains blue and the deoxyribonucleoprotein red. However, the metachromatic volutin granules of the mycobacteria also stained red with the May-Grünwald stain because of their metaphosphate content (Hartman \& Payne, 1954). The fact that the red colour of the granules was not due to their content of deoxyribonucleoprotein was verified by the application of deoxyribonuclease which failed to remove them. The problem of the location of the bacterial nucleus remains to be solved, but the results of this paper show that the electron-opaque granules of the mycobacteria used here are not the nuclei, even though they may be related to the "nuclear apparatus".

The assistance of Miss M. P. Butler, B.A., and of the staffs of the Strangeways and Cavendish Laboratories is gratefully acknowledged. 


\section{REFERENCES}

Bielig, H. J., Kausche, G. A. \& HaArdick, H. (1952). Über den Zusammenhang von TTC-Reduktionsorten und sogenannten Nucleoiden bei Bakterien. Naturwissenschaften, 39, 354.

Brieger, E. M., Cosslett, V. E. \& Glauert, A. M. (1954). Reproductive changes in avian tubercle bacilli studied with the electron microscope. J. gen. Microbiol. $10,294$.

Brieger, E. M. \& Robinow, C. F. (1947). Demonstration of chromatinic structures in avian tubercle bacilli in the early stages of development. J. Hyg., Camb. 45, 413.

Drummond, D. G. (1950). The practice of electron microscopy. J. R. micr. Soc. 70, 27.

Grula, E. A. \& Hartsell, S. E. (1954). Intracellular structures in Caulobacter vibrioides. J. Bact. 68, 498.

Hartman, P. E. \& Payne, J. I. (1954). Direct staining of the two types of nucleoproteins in Escherichia coli. J. Bact. 68, 237.

JAcobson, W. \& WeBb, M. (1952). The two types of nucleoproteins during mitosis. Exp. Cell. Res. 3, 163.

KNAYSI, G. (1951). The structure of the bacterial cell. Bacterial Physiology, p. 28. New York: Academy Press Inc.

Knaysi, G., Hillier, J. \& Fabricant, C. (1950). The cytology of an avian strain of Mycobacterium tuberculosis studied with the electron and light microscopes. J. Bact. 60, 423.

Mudd, S. (1953). The mitochondria of bacteria. In Bacterial Cytology, Symp. 6th Congr. int. Microbiol. p. 67.

Piekarski, G. (1937). Cytologische Untersuchungen an Paratyphus und Colibakterien. Arch. Mikrobiol. 8, 428.

Smith, I. W., Wilkinson, J. F. \& Duguid, J. P. (1954). Volutin production in Aerobacter aerogenes due to nutrient imbalance. J. Bact. 68, 450.

Takeya, K., Koike, M., Uchida, T., Inoue, S. \& Nomiyama, K. (1954). Studies on the nature of granules found in acid-fast bacilli. J. Electron Micr. 2, 29.

Tronnier, E. A. (1953). Zur Existenz des sogenannten Karyoid-Systems bei Corynebacterium diphtheriae. Zbl. Bakt. (1. Abt. Orig.), 159, 213.

WALlHäUSSER, K. H. (1951). Die antibiotischen Beziehungen einer natürlichen Mikroflora. Arch. Mikrobiol. 16, 201.

Weibull, C. (1953). Observations on the staining of Bacillus megaterium with triphenyltetrazolium. J. Bact. 66, 137.

Winder, F. \& Denneny, J. M. (1954). Metaphosphate in mycobacterial metabolism. Nature, Lond. 174, 353.

Winkler, A. (1953). The metachromatic granula of bacteria. In Bacterial Cytology, Symp. 6th Congr. int. Microbiol. p. 39.

\section{EXPLANATION OF PIAATES}

Micro-cultures of Mycobacterium phlei (Söhngen) grown on glycerol agar + embryo extract plates; incubation at $37 \cdot 5^{\circ}$.

\section{Plate 1}

Comparative light- and electron-microscope photographs of impression prints taken on electron microscope grids.

Fig. 1 a. 24 hr. growth stained with Loeffler's methylene blue. The rods and filaments are blue and contain bright red, metachromatic granules (arrows). Light microscope; $\times 2900$.

Fig. $1 b$. Electron micrograph of the same filament as in Fig. 1a. The electron-opaque granules correspond with the metachromatic bodies. Electron microscope; $\times \mathbf{7 2 0 0}$. 
Journal of General Microbiology, Vol. 13, No. 2

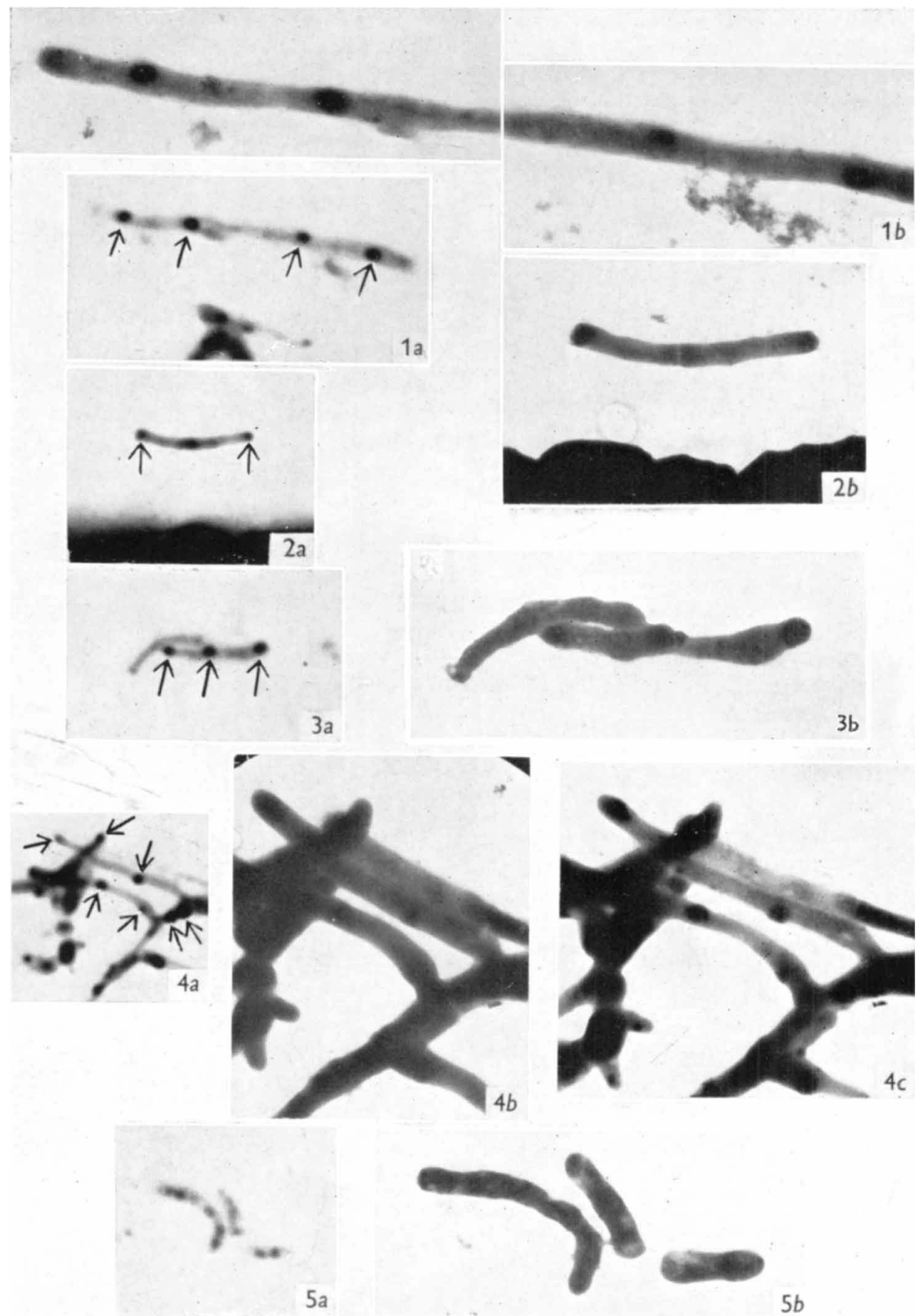

A. M. Glatert \& W. M. Brieger-Electron-dense bonies in M. phlei. Plate, 1 
Journal of General Microbiology, Vol. 13, No. 2
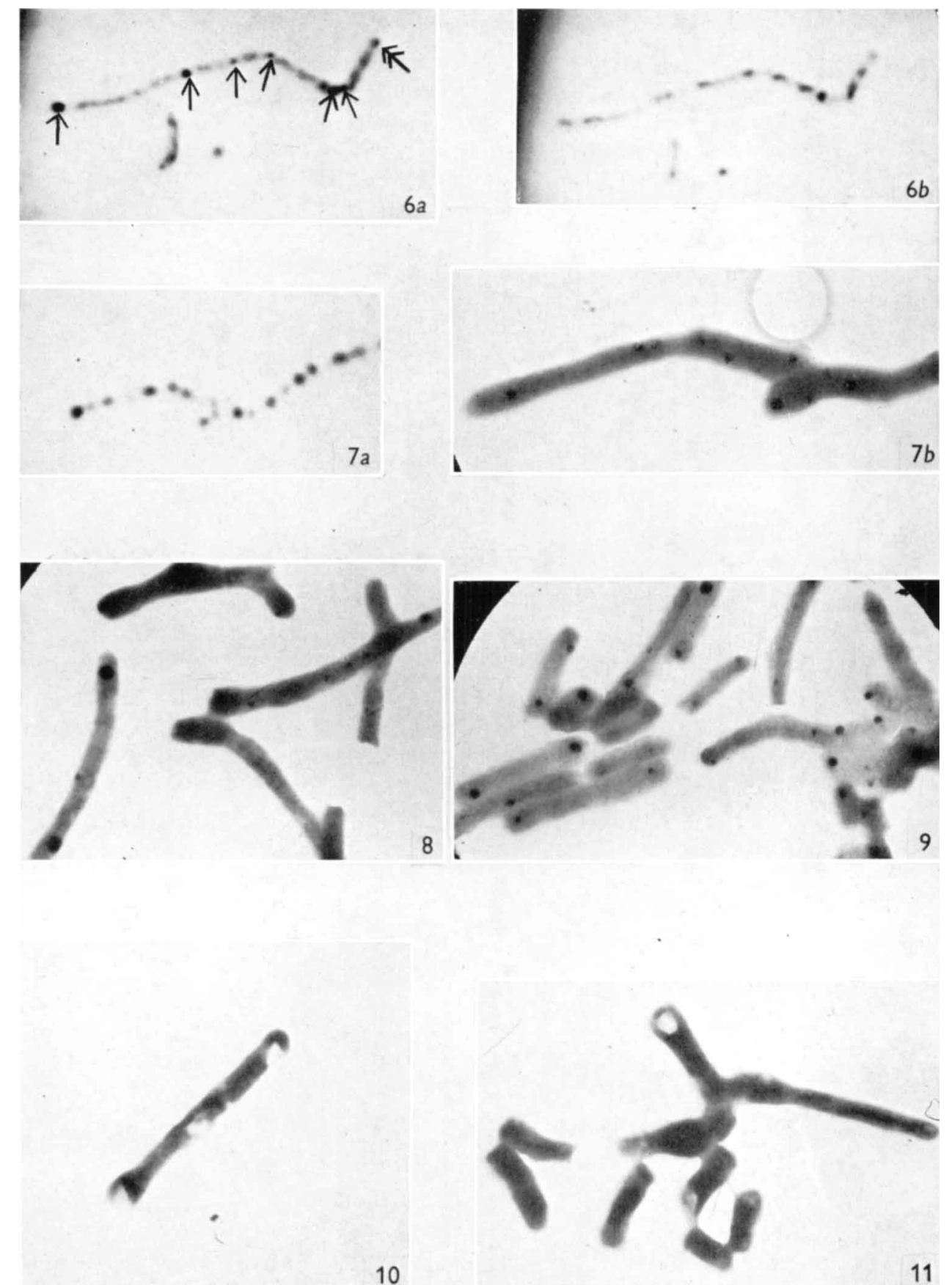

A. M. Glauert \& E. M. Brifger-Ellectron-dense boiles in M. Phlei. Plate 2 
Fig. $2 a .2$ hr. growth stained with Neisser's stain. The volutin granules are marked with arrows. Light microscope; $\times \mathbf{3 1 0 0}$.

Fig. $2 b$. Electron micrograph of the same organism as in Fig. $2 a$. The volutin granules are dense in the electron microscope; $\times 6600$.

Fig. $3 a$. $4 \mathrm{hr}$. growth stained with the May-Grünwald stain. The organisms are blue and contain red granules (arrows). Light microscope; $\times \mathbf{2 5 0 0}$.

Fig. $3 b$. Electron micrograph of the same group of organisms as in Fig. $3 a$. The dense bodies correspond with the red granules of the stained preparation. Electron microscope; $\times 6500$.

Fig. 4a. $6 \mathrm{hr}$. growth stained with May-Grünwald and Giemsa stain. A group of branching filaments, stained blue, with red granular inclusions (arrows). Light microscope; $\times \mathbf{3 4 0 0}$.

Fig. $4 b$. Electron micrograph of the same group of filaments as in Fig. 4a. The density of the cytoplasm masks the internal structures. Electron microscope; $\times 6800$.

Fig. $4 c$. Electron micrograph of the same group of filaments as in fig. $4 a$ and $b$ after electron bombardment in the electron microscope. The cytoplasm is more transparent to electrons and the dense bodies are visible. They correspond to the red granules of the light microscope picture. Electron microscope; $\times 6800$.

Fig. 5 a. $24 \mathrm{hr}$. growth stained with HCl-Giemsa. The organisms contain rows of chromatinic bodies. Light microscope; $\times \mathbf{2 8 0 0}$.

Fig. $5 b$. Electron micrograph of the same organisms as in fig. $5 a$. The Giemsa stain makes the cytoplasm very dense. There is some parallel between the chromatinic bodies of the light microscope picture and the denser parts of this electron micrograph. Electron microscope; $\times 6500$.

\section{Plate 2}

Comparative light- and electron-microscope photographs of impression prints taken on electron microscope grids.

Fig. 6a. 24 hr. growth stained with Loeffler's methylene blue. The red metachromatic granules are marked with arrows. One granule, which appeared red-purple, is marked with a double-headed arrow. The other internal structures are stained blue. Light microscope; $\times 3000$.

Fig. $6 b$. The same filament as in fig. $6 a$ stained with HCl-Giemsa. The chromatinic bodies correspond exactly with the blue structures of the methylene-blue stain. The metachromatic granules are no longer visible, but the red-purple granule, which had an admixture of blue colour, is still present. Light microscope; $\times 3000$.

Fig. $7 a$. $16 \mathrm{hr}$. growth and grown for a further $4 \mathrm{hr}$. on a glucose + phosphate medium containing $0.01 \%$ triphenyltetrazolium chloride. A long filament contains granular deposits of formazan. Light microscope; $\times \mathbf{3 0 0 0}$.

Fig. $7 b$. Electron micrograph of the same filament as in fig. $7 a$. The filament contains some small electron-opaque granules. There is some correspondence between the positions of these dense granules and the formazan deposits, but the dense bodies are much smaller than the stained granules. Also some of the stained granules have no corresponding dense bodies. Electron microscope; $\times \mathbf{7 4 0 0}$.

Electron micrographs of Mycobacterium phlei after various extraction procedures.

Fig. 8. $4 \mathrm{hr}$. growth treated with ribonuclease for $1 \mathrm{hr}$. at $37 \cdot 5^{\circ}$. The electron-opaque granules are still present. $\times \mathbf{7 4 0 0}$.

Fig. 9. $28 \mathrm{hr}$. growth treated with deoxyribonuclease for $1 \mathrm{hr}$. at $37 \cdot 5^{\circ}$. The electron-opaque granules are still present. $\times 7000$.

Fig. 10. $4 \mathrm{hr}$. growth treated with cold perchloric acid. The dense bodies have been extracted. $\times 8100$.

Fig. 11. 28 hr. growth treated with cold trichloroacetic acid. The dense bodies are no longer present. $\times 7800$.

(Received 18 March 1955) 